\title{
Complete resection of undifferentiated cardiac sarcoma and reconstruction of the atria and the superior vena cava: case report
}

\author{
Nobuyuki Furukawa*, Jan Gummert and Jochen Börgermann
}

\begin{abstract}
Primary cardiac tumors are rare with an incidence ranging from $0.001 \%$ to $0.03 \%$ in autopsy series. The prognosis of cardiac sarcomas remains poor because it proliferates rapidly and distant metastases are often found at diagnosis. A 47-year-old male complained of persistent cough. The chest roentgenogram was normal. Subsequent computed tomography revealed a mass in the right atrium. Echocardiography and magnetic resonance imaging confirmed also a right atrial mass $(34 \times 35 \mathrm{~mm}$ ) infiltrating the atrial septum. The tumor was completely resected en bloc, including the anterior and lateral right atrial walls, the left atrial dome, and a large segment of the superior vena cava, and reconstructed the atria and superior vena cava with bovine pericardium. The tumor was histologically and immunohistochemically diagnosed as undifferentiated pleomorphic sarcoma. This type of cardiac sarcoma is very rare and usually found in the left atrium. Twenty-seven months after surgery, the patient is doing well without metastasis or local tumor recurrence.
\end{abstract}

Keywords: Cardiac sarcoma, Primary cardiac tumor

\section{Background}

Primary cardiac tumors are uncommon (incidence in autopsy series, $0.001-0.03 \%)$. Among them, malignant neoplasms account for $<25 \%$ [1]. The prognosis of cardiac sarcomas remains poor. Although a more effective therapy would be desirable, complete resection is the current standard treatment in cases without metastases. We describe a case of undifferentiated pleomorphic sarcoma infiltrating both atria and the superior vena cava. We removed the tumor completely, including the sinus node, by en bloc resection and reconstructed the atria and superior vena cava with bovine pericardium.

\section{Case Presentation}

A 47-year-old male complained of persistent cough. Laboratory tests showed a high CRP level and an elevated erythrocyte sedimentation rate. The patient had a medical history of thalassemia minor and mild anemia but was not taking any medication. Physical examination and chest roentgenogram were unremarkable. Electrocardiography

\footnotetext{
* Correspondence: qtgkk994@yahoo.co.jp

Department of Cardiothoracic Surgery, Heart and Diabetes Center North Rhine-Westphalia, Georgstr. 11, 32545, Bad Oeynhausen, Germany
}

revealed sinus rhythm and incomplete right bundle branch block. Subsequent computed tomography demonstrated a right atrial mass, but no other anomalies. Echocardiography and magnetic resonance imaging confirmed a right atrial mass $(34 \times 35 \mathrm{~mm})$ suspicious for a malignant cardiac tumor infiltrating the atrial septum (Figure 1). The tricuspid valve was intact; right atrial and ventricular sizes were normal; and left ventricular function was good.

The patient was referred to our institution for treatment. The heart was exposed via mid-sternotomy. Because of severe narrowing from the tumor, the superior vena cava was unsuitable as a cannulation site. After full heparinization and flooding of the surgical field with $\mathrm{CO}_{2}$, the ascending aorta, inferior vena cava, and the innominate vein were cannulated. Cardiopulmonary bypass was commenced after the target ACT $\geq 450$ s was reached. A vent was placed into the left ventricle. Exploration revealed that the tumor was penetrating the walls of the right atrium and of the superior vena cava (Figure 2 A). On complete bypass, the ascending aorta was crossclamped and multiple doses of warm blood Calafiore cardioplegic solution were instilled via the aortic root. The tumor was resected en bloc, including the 


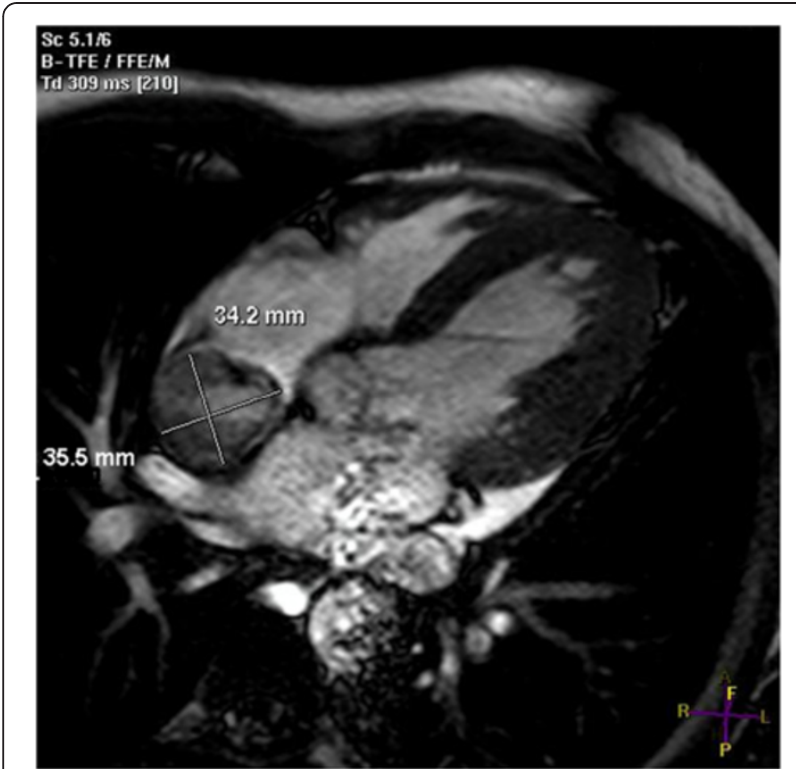

Figure 1 Cardiac magnetic resonance imaging demonstrating a mass in the right atrium involving the left atrial dome and atrial septum.

anterior and lateral right atrial walls (approximately two thirds of the right atrium altogether), the left atrial dome, and a large segment of the proximal superior vena cava (Figure $2 \mathrm{~B}$ ). The atrial septum was found to be free. The left atrial roof was reconstructed with a patch of bovine pericardium (Figure $2 \mathrm{C}$ ) secured with running 4-0 prolene (Ethicon, Norderstedt, Germany). The right atrium and the superior vena cava were each reconstructed with a folded strip of bovine pericardium, again using running 4-0 prolene suture, making sure that the neo-right atrial dome provided sufficient reservoir capacity (Figure 2D). The preliminary histologic evaluation revealed clear margins. The aortic clamp was removed after careful deairing. As expected, the heart was beating in a nodal escape rhythm because the sinus node had been removed. We therefore secured permanent bipolar epicardial pacemaker leads to the remaining right atrium and to the left ventricle. The leads were brought out through the inner layers of the thoracic wall and connected to a DDD pacemaker which was implanted in the left pectoral area. Under mild inotropic support, the patient was easily weaned from cardiopulmonary bypass. All cannulas were removed and the cannulation sites oversewn. Protamin was administered. The pericardium was loosely approximated over a drain. The wound was closed in layers after careful hemostasis. The patient was transferred to the intensive care unit in stable condition.

The patient recovered without complications, was discharged home on postoperative day 7 and referred to undergo local radiotherapy and systemic chemotherapy with ifosfamide and paclitaxel. For a duration of three months, the patient was anticoagulated with warfarin and then switched to oral acetyl salicylic acid. Thirty-five months after surgery, the patient is doing well and remains free of metastases or local tumor recurrence.

Histological examination (Figure 3) revealed dense groups of polygonal and spindle cells with eosinophilic

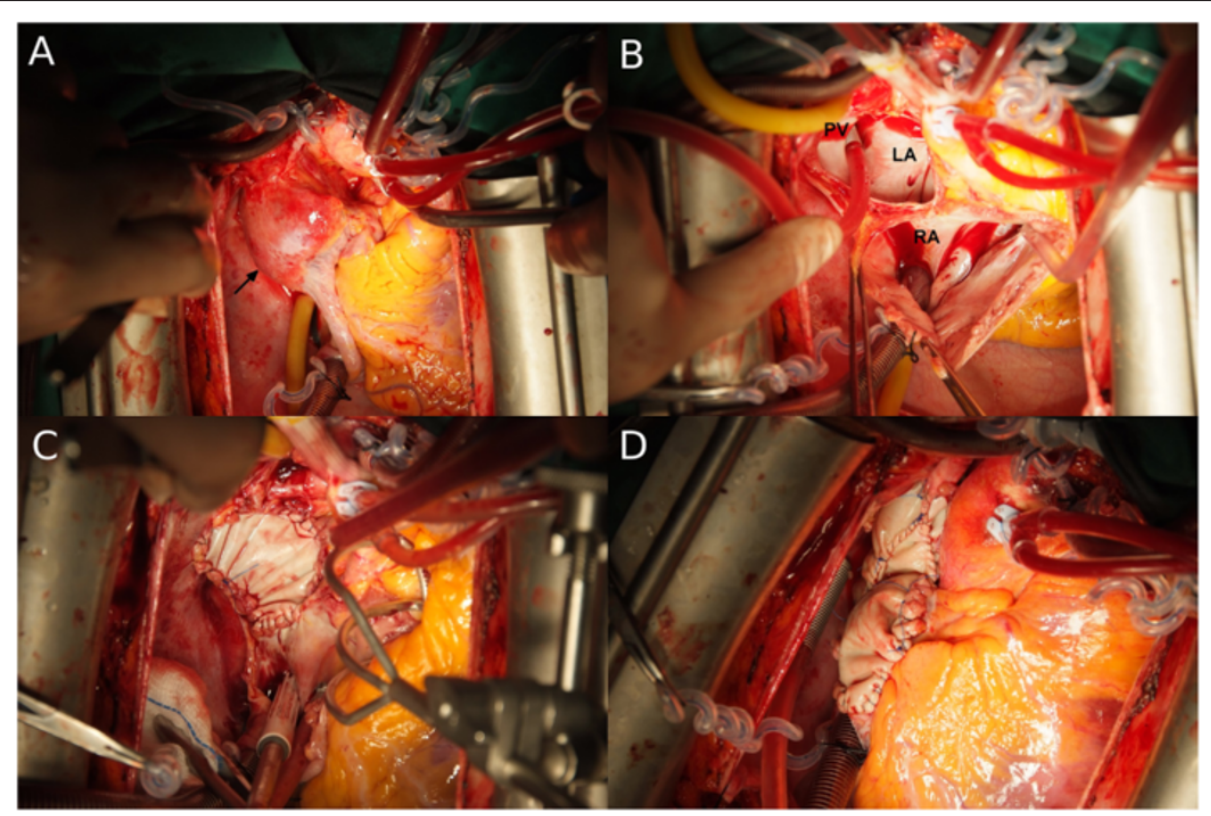

Figure 2 Intraoperative view. Note that the tumor extends into an through the wall of the superior vena cava and the left atrial dome (A). Complete tumor removal by en bloc resection, including the right atrial anterior and lateral wall, left atrial dome, and a large segment of the superior vena cava (B). Repair of the left atrial dome (C). Reconstruction of the right atrium and superior vena cava with bovine pericardium (D). 
cytoplasm and large pleomorphic nuclei with high density chromatin and irregular nucleoli. On immunohistochemical examination, the neoplastic cells stained positive for vimentin but negative for pan-keratin, desmin, actin, and histiocyte markers. These observations are compatible with a histological diagnosis of undifferentiated pleomorphic sarcoma.

\section{Discussion}

Primary cardiac tumors are uncommon. Approximately three fourth of them are malignant [1]. Sarcomas comprise two-thirds of these malignancies [2]. Cardiac sarcomas include angiosarcoma; sarcoma with myofibroblastic differentiation; synovial sarcoma; and rhabdomyosarcoma. Sarcomas with myofibroblastic differentiation are the most diverse types, subclassified as undifferentiated pleomorphic sarcoma; osteosarcoma; leiomyosarcoma; and fibrosarcoma [3]. In several large series [4,5], the most common type was angiosarcoma, while undifferentiated pleomorphic sarcomas were relatively rare (only 6-7\%). Undifferentiated pleomorphic sarcoma is usually found in the left atrium and exhibits a solid and infiltrative pattern. As described here, undifferentiated pleomorphic sarcoma with bilateral involvement is very rare [6].

The prognosis of patients with cardiac sarcoma is poor because the tumor proliferates rapidly and distant metastases are often found at the time of the initial diagnosis. Hamidi et al. reported that the median survival of patients who underwent surgery was 12 months, whereas survival of patients not operated upon was only 1 month [1]. Simpson et al. reported that the median survival of patients with and without metastasis was 5 and 15 months, respectively [4].

In our case, the tumor infiltrated the left atrium and approximately two-thirds of the right atrium, involving the sinus node and superior vena cava. Fortunately, this infiltration was relatively anterior in the left atrial dome, facilitating complete resection and repair with bovine pericardium. When the tumor infiltrates the left atrium more deeply and to a greater extent, resection and reconstruction is relatively difficult. In such cases, autotransplantation is an option permitting complete resection and repair $[2,7]$.

Cardiac sarcomas are often asymptomatic. In advanced cases, they occasionally produce nonspecific symptoms. Malignant cardiac tumors with direct invasion of the pericardium can cause pericardial effusion or arrhythmias, such as atrial fibrillation, heart block, or recurrent ventricular tachycardia [2,3]. We performed en-bloc resection of the tumor, including the sinus node. Simultaneously, permanent epicardial pacemaker leads were placed onto the remaining right atrium and onto the left ventricle. Radical resection of the tumor is difficult or impossible if the tumor infiltrates the conduction system [8]. In many cases, cardiac sarcoma is so widespread that only palliative surgery can be performed [8]. It remains unclear if there is an alternative to radical surgical resection in patients without metastatic disease. Because cardiac sarcoma is uncommon and because there are only a few limited studies on the subject, no standard treatment has been defined. Although aggressive resection of cardiac sarcoma is challenging, some studies clearly demonstrate that complete tumor removal is the best method for improving survival $[1,2,4]$.

In our case, chemotherapy and radiotherapy were initiated relatively early after surgery, especially in view of reports [2,7] of local recurrences or distant metastases several years after successful resection. The effectiveness of such adjuvant therapies for cardiac sarcoma remains controversial. However, studies [1,2] have shown that combining several carcinostatics is more effective than single-agent therapy, and that postoperative radiotherapy can improve survival.

\section{Conclusion}

We presented a rare case of undifferentiated pleomorphic cardiac sarcoma successfully removed by enbloc resection which included two-thirds of the right

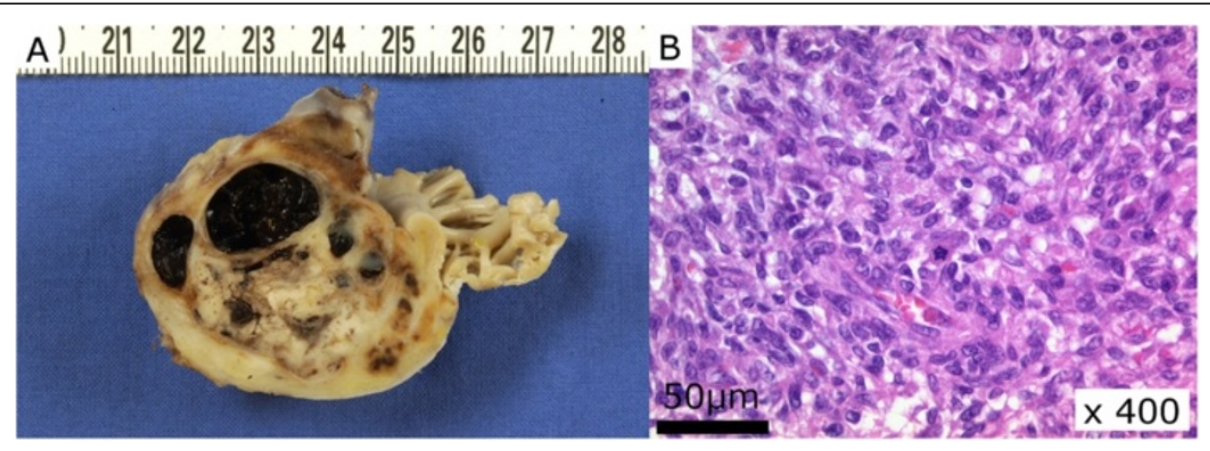

Figure 3 Cut surface of the lesion showing the pale yellow and solid part of the tumor and the cystic component which is filled with coagulated blood. (A) An HE-stained microscopic section reveals dense groups of polygonal and spindle cells with eosinophilic cytoplasm and large pleomorphic nuclei with high density chromatin and irregular nucleoli (B). 
atrium, the left atrial dome and a large segment of the superior vena cava, reconstructing the defects with bovine pericardium. Despite successful management, close follow-up is mandatory because of the overall poor prognosis of cardiac sarcoma.

\section{Consent}

Written informed consent was obtained from the patient for publication of this case report and any accompanying images. A copy of the written consent is available for review by the Editor-in-Chief of this journal.

\section{Competing interests}

The authors declare that they have no competing interests.

\section{Authors' contributions}

NF carried out the manuscript and collected references. JB and JG helped to revise the manuscript. JB and JG underwent the operation. All authors read and approved the final manuscript.

Received: 8 May 2012 Accepted: 23 September 2012

Published: 27 September 2012

\section{References}

1. Hamidi M, Moody JS, Weigel TL, Kozak KR: Primary cardiac sarcoma. Ann Thorac Surg 2010, 90:176-181.

2. Bakaeen FG, Jaroszewski DE, Rice DC, Walsh GL, Vaporciyan AA, Swisher SS, Benjamin R, Blackmon S, Reardon MJ: Outcomes after surgical resection of cardiac sarcoma in the multimodality treatment era. J Thorac Cardiovasc Surg 2009, 137:1454-1460,

3. Burke A, Jeudy J Jr, Virmani R: Cardiac tumors: an update. Heart 2008, 94:117-123.

4. Simpson L, Kumar SK, Okuno SH, Schaff HV, Porrata LF, Buckner JC, Moynihan TJ: Malignant primary cardiac tumors: review of a single institution experience. Cancer 2008, 112:2440-2446.

5. Putnam JB Jr, Sweeney MS, Colon R, Lanza LA, Frazier OH, Cooley DA: Primary cardiac sarcomas. Ann Thorac Surg 1991, 51:906-910.

6. Orlandi A, Ferlosio A, Dell'Anna V, Quitadamo R, Pellegrino A, Spagnoli LG: Undifferentiated sarcoma of the heart: a rare clinicopathologic presentation. J Thorac Cardiovasc Surg 2002, 124:192-193.

7. Doty DB, Doty JR, Reid BB, Anderson JL: Left atrial sarcoma: resection and repair by cardiac autotransplant and in situ pericardial patch. Ann Thorac Surg 2006, 82:1514-1517.

8. Brunner-La Rocca HP, Vogt PR, Burke AP, Schneider J, Jenni R, Turina MI: A primary cardiac sarcoma with unusual histology and clinical course. Ann Thorac Surg 2001, 71:1675-1677.

doi:10.1186/1749-8090-7-96

Cite this article as: Furukawa et al:: Complete resection of undifferentiated cardiac sarcoma and reconstruction of the atria and the superior vena cava: case report. Journal of Cardiothoracic Surgery 2012 7:96.

\section{Submit your next manuscript to BioMed Central and take full advantage of:}

- Convenient online submission

- Thorough peer review

- No space constraints or color figure charges

- Immediate publication on acceptance

- Inclusion in PubMed, CAS, Scopus and Google Scholar

- Research which is freely available for redistribution 